\title{
Transcendence: Can live performance art in combination with interactive technology induce altered states of consciousness?
}

\author{
Maria Almena \\ Kimatica Studio, Flux Events \& \\ Kinetica Museum \\ 4 Nettleton Road \\ London SE14 5UJ, UK \\ maria@kimatica.net
}

\begin{abstract}
The main focus of this paper is to explore the re-contextualisation of the concept of transcendence as an altered state of experience within digital art performances. Is there a transcendental art movement in the contemporary digital art scene and does it exist in response to the lack of emotional and spiritual connection in general Western Society? How could we use our contemporary mediums, tools and techniques to induce an altered state that can have beneficial effects for artists, audiences and our society as a whole?
\end{abstract}

Transcendence. Altered states. Digital performances. Ritual art. Shamanism. Audiovisual experiences. Consciousness.

\section{INTRODUCTION}

Altered states of consciousness (ASCs) have been pursued by humans consistently throughout our known history. From ancient shamanic rituals to the pervasive presence of psychedelia in modern culture and the Arts, the cultural attraction of transcendental experiences is undeniable. However, only recently, there has been a resurgence, mainly through transpersonal psychotherapy and neuroscience research, in attempts to better understand these processes. What causes them? What is their effect on humans? And could they have a positive influence?

We will be exploring transcendental experiences within the art world. The term "transcendental" was coined by Immanuel Kant through his doctrine "transcendental idealism", describing conditions of possibility of knowledge. Transcendent meaning is that which goes beyond usual knowledge of human being.

Combining scientific, anthropological and psychological research with visual arts, interactive technology and live performance, Transcendence by Kimatica, will explore how to create an immersive experience that will attempt to induce ASCs in its audience. A multidisciplinary and collaborative research project aiming to answer to what degree biochemical modification is required to alter consciousness and whether ritualistic, hallucinogenic spaces can be created through external, sensory stimulus alone.

This practice-lead research will also explore the relationship between digital performance and shamanic rituals researching the benefits that this could have in our contemporary society, mainly through the work of Kimatica. We will also be exploring other contemporary media artists that intend to reach beyond the known, creating installations and performances that elevate. Transcending knowledge and experience that escapes our traditional senses, aiming for the margins of human consciousness.

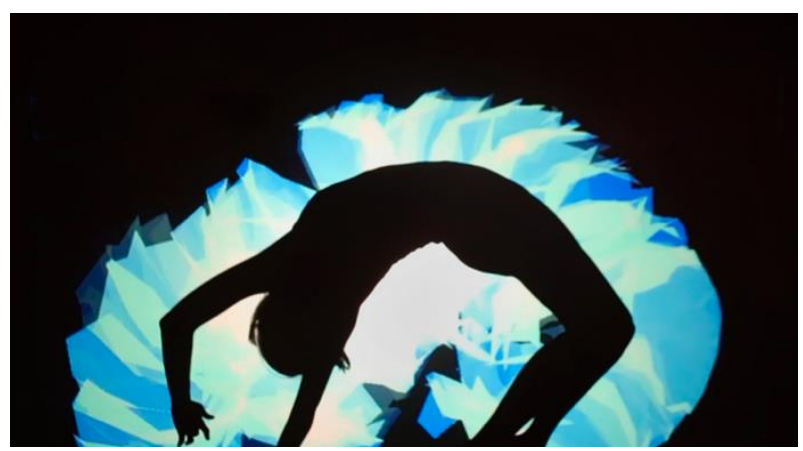

Figure 1: Visual stimulus: previous work by Kimatica. 

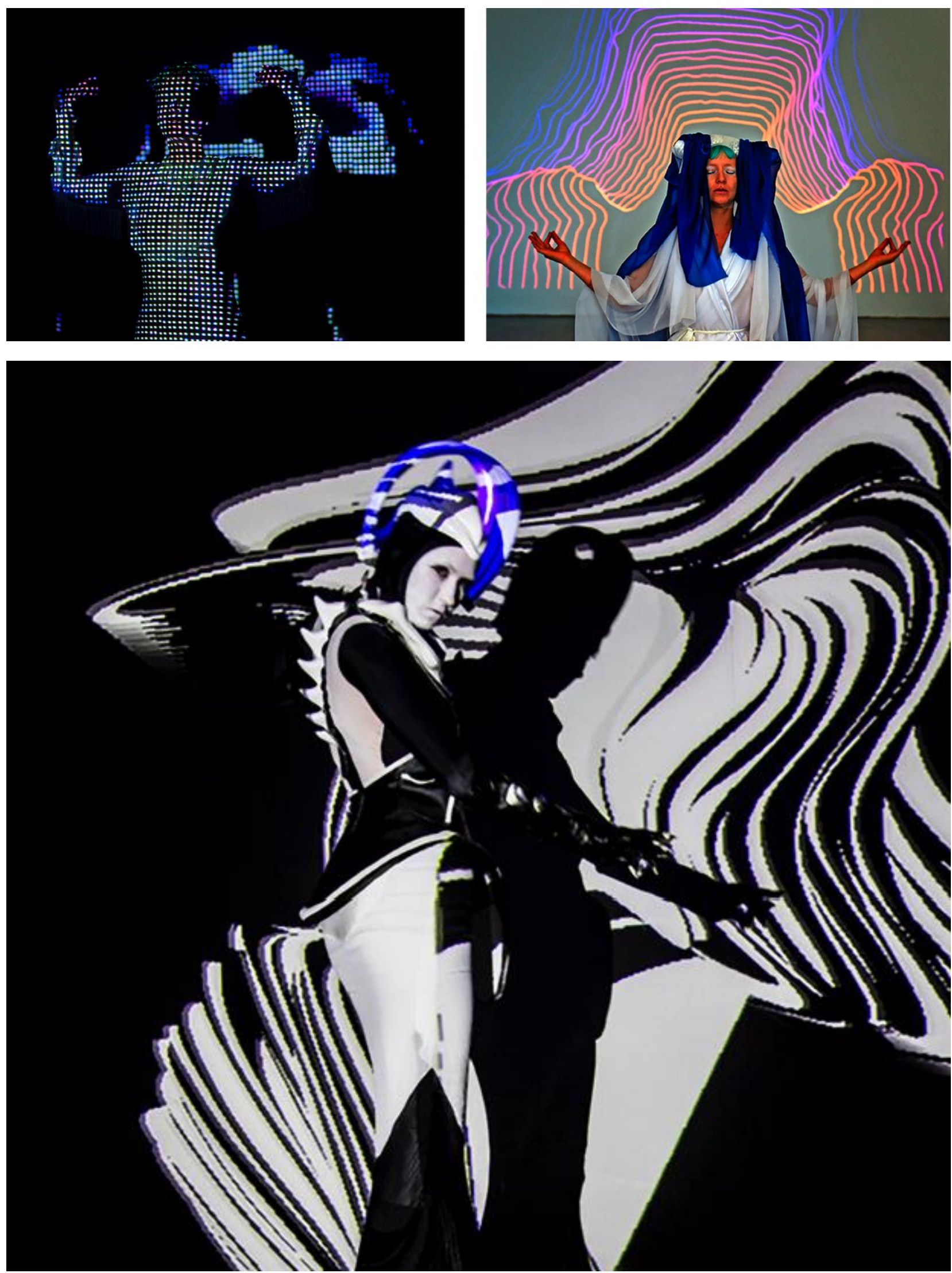

Figure 2: Visual stimulus: previous work by Kimatica Studio. 

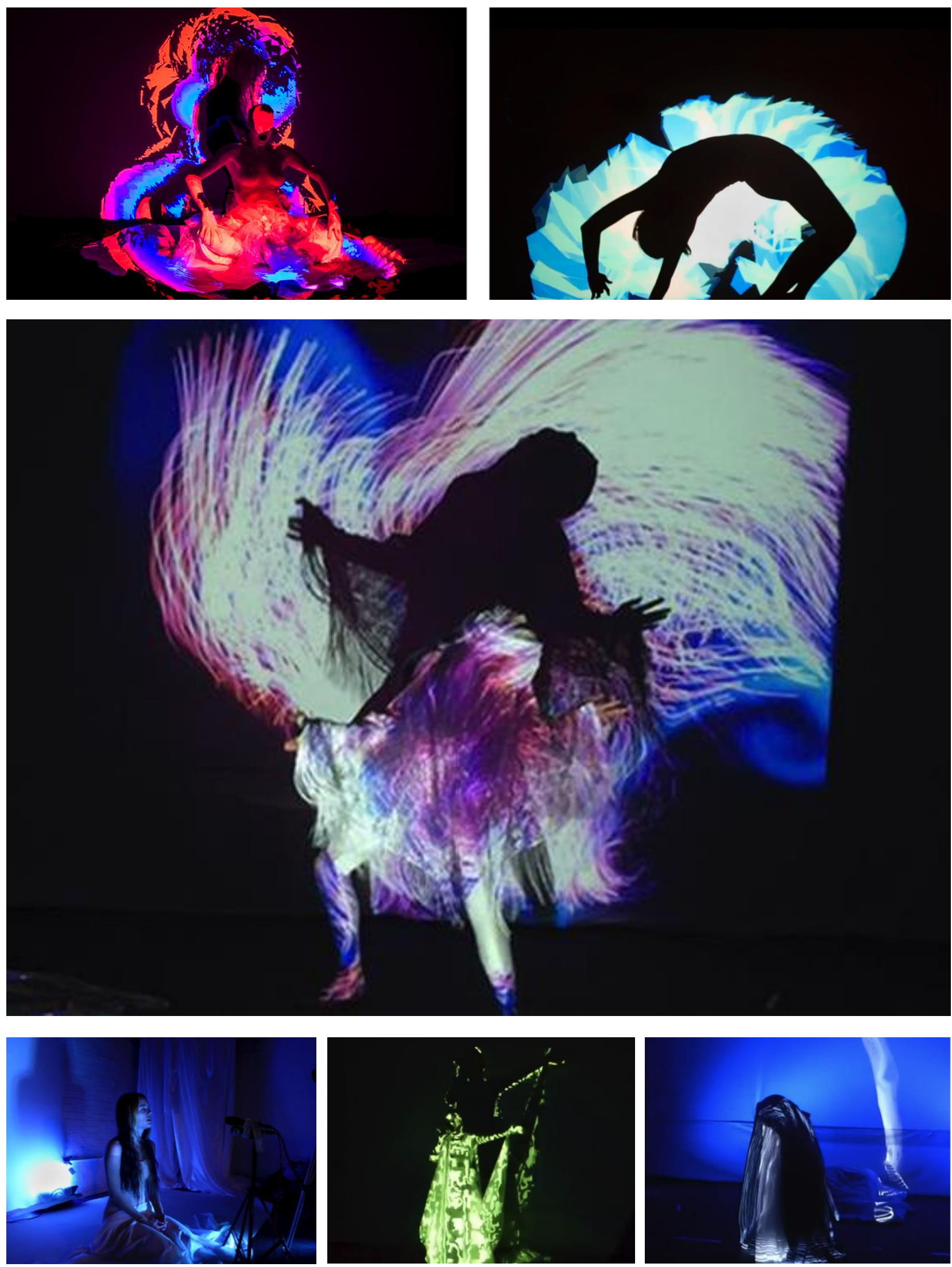

Figure 3: Visual stimulus: previous work by Kimatica Studio. 


\section{TRANSCENDENCE BY KIMATICA}

This is a preliminary study to explore the research available on altered states and the concept of transcendence within the arts, shamanism, ritual and performance art, interactive technology and light and sound therapy. Also to relate this academic research to our practice-lead research to be used in the production of our new live digital performance: Transcendence.

The artist's task is to save the soul of mankind; artists who are self-selected for being able to journey into the Other, if the artists cannot find the way, then the way cannot be found...What the computer and the virtual technologies all push toward is to bring the soul into visible manifestation - Terence McKenna, 1999.

\subsection{Altered states and transcendence in the arts}

An altered state of consciousness (ASC) may be defined as a temporary change in the overall pattern of subjective experience, such that the individual believes that his or her mental functioning is distinctly different from certain general norms of his or her normal waking state of consciousness (Tart 1990). An ASC separates the experience of the body from the experience of the mind, whereas the mental and physical separation between the perceiver and what is perceived are clear in someone who is awake. During an altered state, such as a trance, hallucinatory, meditation or transpersonal experience, the cultural line between the two worlds disappears and time will be distorted while the experience takes place (Tart 2008).

The aim of this research is to use altered states of consciousness (ASCs) as an adaptive principle for creating an original digital performance by Kimatica, in which common features of the ASC experience provide a basis for the design of the visual, sonic and physical material, and inform the structural aesthetic and theory corresponding to the different performance sections (Weinel 2012).

According to Farthing, there are fourteen dimensions of changed subjective experience, which characterise ASCs. Namely: attention, perception, imagery and fantasy, inner speech, memory, higher-level thought processes, meaning or significance of experiences, time experience, emotional feeling and expression, level of arousal, self-control, suggestibility, body image, and sense of personal identity. We will be looking at exploring some of these concepts in our live research and experiential testing with artists and audience.

Trance state has been defined as: a condition of dissociation, characterised by the lack of voluntary movement, and frequently by automatism in act and thought, illustrated by hypnotic and mediumistic conditions (Lewis 1989, p.33).

The 'transcendental sublime' explores how a work of art can enable one to be transported, going beyond the given limits to a place of accessing one's spiritual side. It brings back a Romanticism approach, a movement, which emphasised intense emotion as an authentic source of aesthetic experience. Some artists today are using contemporary media and technology to create experiences that take their viewers into new places or states. For example, James Turrell (2002) works with light and how it fills a space in order to create an experience of wordless thought.

Advances in science can even measure the intensity of the meditative experiences art can create. Scans have pinpointed which areas of our brains go into high gear and which become dormant when we have such an experience. This kind of new techniques of measuring the impact are very useful for our research and we are intending to look at the artistic and psychological impact, as well as the neurological impact, on the performers, artists and audience so that we can obtain an overview on the impact of our experience.

Sometimes it feels like artists point the way to aspects of the universe that cynics have lost sight of (Mark Levy 1993).

\subsection{Shamanism, ritual and performance art}

Shamanism represents the most widespread and ancient methodological system of mind-body healing known to humanity, and has utilised methods to produce altered states of consciousness in themselves and their patients for thousands of years. The shaman moves between realities, they are the middleman between the conscious and the subconscious world, or the ordinary reality and the non-ordinary reality (Michael Harner 1990).

Many rituals, from past primitive cultures to the modern civilised world, involve the use of altered states. Different ways of achieving ASCs have been used over the years and include hallucinatory drugs, meditation, hypnosis, mantras, drumming, dance trance, sex and pain (Farthing 1992).

Some artists have resumed the ancient role of the shaman "the technician of ecstasy" (Eliade 1964). Like shamans, many artists have enhanced powers of seeing, hearing and dreaming, and move easily between different realities. Shamanic-artists are spiritual practitioners who use creative processes to engage the unconscious, archetypes, divinity, collective unconscious, paranormal realms and 
entities, the inner world and experiences of the sacred.

European artists have embraced shamanic thinking through different periods of time (Hoppál 1996). One of the most important antecedents of contemporary performance artists employing shamanistic practises is the Dadaist poet Hugo Ball, creating live art that aimed to connect with the inner world, creating irrational, primitive and complex work. He also explored the power of poetry as a mantra to enter into other states, a technique we would be exploring within our sound design.

Another iconic example is Joseph Beuys, a selfidentified shaman, who created ritual performances and objects with the intent to heal. He wanted to sculpt the consciousness of this students and spectators exploring the idea of energy exchange between audience and performer. He used repetitive gestures and/or sounds over a lengthy time period to put his audience into a trance state, an approach to performance arts that is very relevant to our research.

There are plenty of questions and a lot of research to do about how to translate these concepts of ancient ritual, shamanism and trance into a contemporary performative piece. But something we can use as a starting point from psychology theories is that we are empathic animals and we feel what others feel, so when someone attains a trance or goes through a powerful transformation, this can be transferred (Bradford 2006).

Eberhard Sheiffelei (2001) is exploring how performers get into transcendental states through their acting. He believes during the performance, actors are more connected to the meditative state of the now than people in ordinary states.

Some artists who explore performance art as contemporary rituals do get into a trance in their pieces, they can lose all concept of time and feeling, they can even feel the energy shifting within themselves and try to transfer this to the audience. One of the artists who explores this in her performances is Louis Flleishauer (Jose Sanchez Rivas 2017) As well as some of the performance by Marina Abramovic such as "Ritual, repetition and mourning" or "Rituals of breath, voice and void".

The big question I encounter in this research is, how an artistic experience can be as transformative as a shamanic ritual? So far my research leads me to think that it needs to be a real experience, so the performance piece has to become a real ritual and the whole experience has to be taken as true sacred experience like shamanic rituals do.

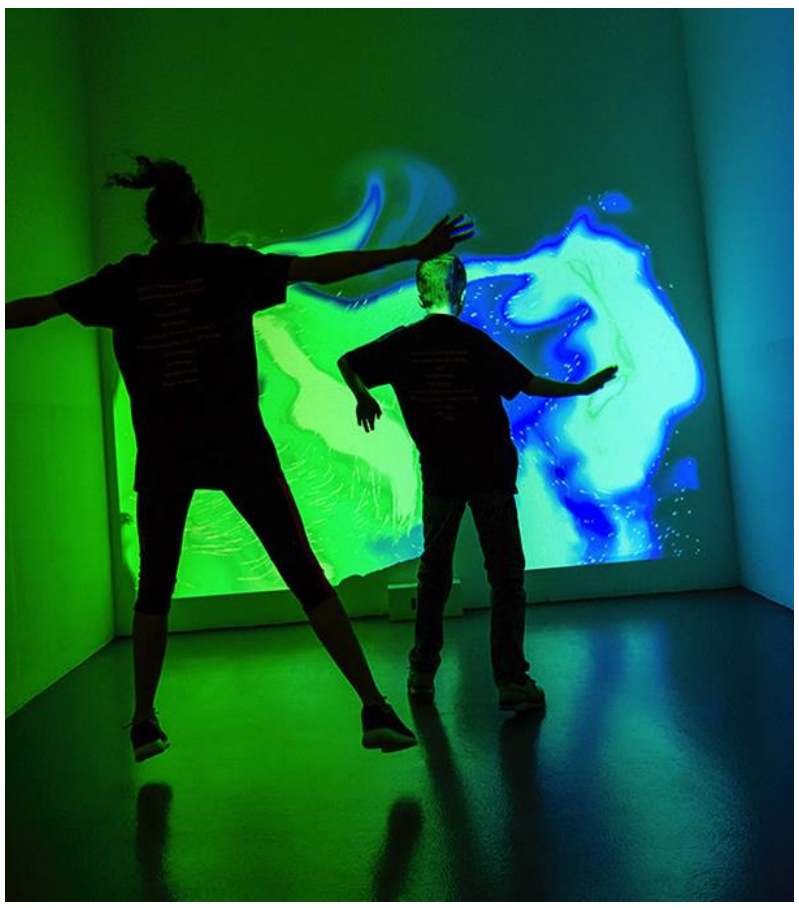

Figure 4: Visual stimulus: previous work by Kimatica.

It is also essential to convert theatre spaces into sacred spaces where meaningful rituals and transformative changes can occur. In ancient Greek theatre, they prepared the space for a ritual performance with the purification of the stage before a new piece by killing an animal and using its blood Mark Pizzato (2002).

To create a performance that aims to transform, enlighten, and hopefully heal and empower the artists involved in the project and the audience watching, all of these sacred and ritualistic elements are to be taken into account.

\subsection{Interactive technology, light therapy, sound and altered states}

Kimatica's main research focus is on interactive technology, light therapy and sound, particularly, how these mediums can help to trigger altered states within a contemporary performance art ritual.

One of the first attempts to explore light therapy within an artistic context was the dream machines from Brion Gysin (1973), which is a stroboscopic flicker device that produces visual stimuli. It is "viewed" with the eyes closed: the pulsating light stimulates the optic nerve and thus alters the brain's electrical oscillations. In 1998 a more advanced version of the dream machine was created, Pandora. A lighting device, which uses a similar technique, also stimulating various brain regions by focusing rays of light on the retinas. Through closed eyelids, users experience a "visible" frequency entrainment of kaleidoscopic patterns of colours and psychedelic imagery, which 
provides an immersive environment for deep level brainwave entrainment. The highly programmable software included with the device allows you to control the flickering light in dynamic ways.

One artist and neuroscientist who has been exploring light therapy within the arts is Luciana Hail. She has developed the use of Pandora into a series of performances and installations involving the real time monitoring and sonification of the participants own brain waves using techniques from hypnosis and meditation.

Research by Dr. Herbert Benson (Harvard) in trance and repetition has demonstrated that almost any "neutral" word repeated silently to oneself generates some of the benefits documented with meditative mantras. The aim of Jose Macabra, Transcendence sound designer, is to represent or induce altered states of consciousness through Sound Design to accompany the performance piece and portray a clear representation of the inner world.

A vast range of sound manipulation, composition, music and spatialisation techniques will be used to build Transcendence sonic material that will take the audience into a journey between the conscious and unconscious realm. To achieve these results, techniques such as the use of organic sounds, transformation of recordings, sound shortening /stretching, built of complex entoptic rhythm sequences, automatic and Dadaist approaches to composition, sound poetry, vocal processing, improvised sound, synthesis and link with the sound and the visual, changes in pitch, harmony and the use of timeless drones.

A combination of analogue and digital software and hardware will help with this task, to make the sound a fundamental part of the vehicle for this journey. Noise and electro-acoustic music will be the main influences when compromising to an aesthetic as this composition is building up from rural to urban sounds as well as near future. Noise is irrational and will play an important role when describing ASCs and position it in the composition it a battle with the rational order of harmonies and melodies music. The representation of the spirit world and the blend with reality observed often during ASCs is the main objective, presenting us with a way of leading or constructing parallel realities through light and sound, reinforcing a strong link with Shamanic culture and the origin of art portraying ASCs. Ecstasy and Trance will be feeding on the audience to performers and vice versa.

A contemporary art group who is exploring interactive art through similar mediums is Anemala Group. They create experiences on the intersection between art and technology intending to reflect on the nature of perception, exploring the relationships between sound, colour, light, movement and form. Their focus in participatory art fills the gap between performers and audiences, enabling them to question their senses and the boundaries of perception.

For Transcendence, we will be addressing all theses theories in a digital performative context. One of the techniques we will be exploring is the overstimulation and hyper-stimulation of the senses, through light and sound, to be in the meditative state of the now. One of the biggest and most difficult ASC for our current society is to be present, so therefore we believe it could be one of the most beneficial to research and aim to achieve.

\subsection{Digital performances, transcendental art and interactive technology}

Ancient Greek theatres employed elaborate mechanical stage devices to amplify the gestures of the performers allowing them to portray gods with superhuman powers. We'll be exploring similar effects with Transcendence but using the interactive technology available today.

Nowadays the use of digital technologies has resulted in an experience of multiple hyper-realities and multi-directional processes helping to represent the complexity of the different layers of the perception and human consciousness. (Susana Broadhurst 2006).

One of the available technologies that we use the most is motion tracking. This technology enables the generation of 3D visuals that respond to dancers' motion and vice versa - the visuals themselves, in turn, also enhance their movement. Carol Brown, a digital performance artist, for example, investigates new characteristics of dance and performance art arising from an intertwinement of the embodied and the informational in environments in which the boundary between the real and the virtual is blurred (Susana Broadhurst 2006).

This tracking technology allows us to use the information obtained by computer vision analysis as input for the algorithms that generate the visuals and the visuals are affected by the gestures of the performer. This interactivity adds another layer of expressiveness and "liveness", making every performance unique and unrepeatable.

There is a close relationship between this way of creating effects and the way magicians or shamans create their effects. So we are intending to explore this way of ritualisation using the relation between light \& sound, body, movement and their way to trigger emotions. Aiming to create an intimate 
relationship between the body and movement, with sound and lighting.

These techniques allow us to affect different aspects of the performance experience, which then affects the audience's perception of the piece, and we believe this might lead to the creation of altered states of consciousness. The projected visuals create a second digital skin that augments the body and its surroundings, an augmentation of the expressiveness of the movement of the performer.

The types of visual effects we use are mainly generative and as such rendered in real time. Most of these effects can't exist without a subject in front of the camera sensor. Our visuals heavily rely on the $3 d$ data received from a sensor that encodes the position and movement of the subject. They highlight the relationship of the performer with the space and react to the changes in position and speed. Our aim is to create abstract visual patterns that are consistent with the physical space but that alter it's meaning. Most of the abstract patterns that we use are the result of an interaction between the physical presence and movement of the performer and computer algorithms that analyse and render spatial and temporal data.

Other artists who have been exploring how technology can be achieved as a transformative tool are Cyberdelics Incubator. They showcase, review and discuss perceptual technologies that have the potential to expand our experience of ourselves and the reality we live in. They explore a range of cyberdelic concepts and open the discussion on the various aspects of how we can create transformative experiences within these immersive technologies. They and other contemporary digital artists such as Chris Levine, Kito Mbiango or Krista Kim with her Techism movement, show how this transcendental art movement in the digital arts is a currently existing movement.

\section{PRACTICE-LED RESEARCH}

This enquiry investigates the subject through an iterative mode of led-practice research. We created two performance pieces in 2012 and 2014, where we started to research these concepts: Contemporary Ritual (https://vimeo.com/53073221) and Butho (https://vimeo.com/122999864).

During 2015 and 2016 we focused on research, and in 2017 we started working towards creating our first sketch for Transcendence live research at Breaking Convention conference, a multidisciplinary festival exploring concepts of the psyche, in the University of Greenwich

(https://vimeo.com/235161798/ec32fa160f).
We thought this kind of event would be the perfect place to test the first stage of our research. David Luke, Doctor in Psychology and director of this conference, thought our performance was bringing the subconscious mind into a performative experience to take the audience back to the subconscious mind. We had some very deep insight regarding the experience from the audience (https://vimeo.com/26094480).

This year we have planned several experiential tests with psychology and neuroscience students to complement our artistic practical research. These experiential tests will quantify and measure the psychological and emotional impact on participants (the audience) of a series of experiences that are aimed at manipulating perception through the use of light, sound and physical movement. The data of the research will help to understand better which external sensory stimulus can facilitate euphoric states during spectatorship and this can be applied to different wellbeing, psychological and artistic projects.

We will be addressing these research subjects:

- Perception (visual, scent, touch and acoustic)

- Manipulation through the use of technology.

- Sound spatialisation and psychoacoustics.

- How to exploit certain optical and acoustic illusions to replicate the perceptive state of altered states of consciousness.

- Layering manipulated light, sound and movement over the conventional perceived reality to transform the meaning of the scene.

- Alteration of human senses through a guided multi-sensorial experience.

- Researching traditional altered states techniques (shamanic rituals, hypnosis, meditation, dance trance) and incorporate this methodology into a contemporary approach.

- Explore how to apply these transcendental experiences in a therapeutic/holistic approach for well being, such as helping people to meditate.

Our next live showcase is in Splice festival, Rich mix 12th of May 2018, London. Here we will put into practice our experiential tests and academic research so far.

\section{CONCLUSION}

Most artists create art with the intention to benefit others. In order to try to achieve this, the audience is positioned on the forefront of the intention when 
creating new work, especially in the interactive art. This is why artists interested in the therapeutic use of the arts, have to spend much more time analysing their audience if they are to create a real impact.

Exploring the audience relation with performances is vital to create a strong and transformative ritualistic experience. This is why it is very important to create a safe space to allow the performers and the audience to equally have deep inner experience. Creating a cohesive collective experience or group ritual will help to enhance this relationship.

Researching the way in which energy exchanges between the audience and the performers happens is going to be key to evolve in this subject. Performers and artists will need to explore how to get into trance and enter the ASC by themselves. Only in this way they can inspire the audience to do the same.

We are aiming for this practice-led research to lead to publication, more performances and broad collaboration between disciples, community and group engagement. All of this with the intention to create a methodology that would allow us to navigate between the different states of consciousness or perception through a participatory artistic experience.

We want to create a novel research protocol that can be used by other audiovisual and performance artists or enterprises, who want to create live immersive experiences researching similar themes. The data from the research will help to understand better which external sensory stimulus can facilitate transcendental states and this can be applied to different well being, psychological and artistic projects.

We are going to explore how to measure the impact on audiences. Carl Smith, Director of the Learning Research Centre at Ravensbourne, who specialises in the use of technology to achieve ASC, will be assisting us with specific software and hardware that will help us to analyse the impact. Ravensbourne are developing a Sensor Fusion wearable garment that can be worn by audience members to measure changes in affective state. The wearable has a mix of hard and soft biosensors to sense changes in heart rate, posture and Galvanic Skin Response (GSR).

So far this research establishes that altered states of consciousness, achieved through either religious ritual or medical practice, have been both historically and socially accepted and seen to have a beneficial impact, so Transcendence as a multidisciplinary art-tech-science collaborative research will expand upon this reflections aiming to replicate this experience, but with our own unique artistic approach.

In the words of Marina Abramovic (2010):

Artists today? They are couriers; they accompany people on the true adventure, a journey into the inner self. Artists accompany us on our search for a new order.

\section{REFERENCES}

Abrahan, R., Mckenna, T., and Sheldrake, R. (2001) Chaos, Creativity, and Cosmic Consciousness. Park Street Press, Maine, USA.

Abramovic, M. (2017) Inside Out MoMA PS1 Blog. https://www.moma.org/explore/inside out/2010/06/03/ma rina-abramovic-the-artist-speaks (retrieved 21 September 2017).

Benyshek, D. (2018) Artists as Shamans: Historical Review and Recent Theoretical Model. http://www.academia.edu/.../Artists as Shamans Histori cal Review and Recent Theoretical Model (retrieved 7 April 2018).

Broadhurst, S., and Machon, J. (2006) Performance and Technology. Practice of Virtual Embodiment and Interactivity.

Cyberdelics Incubator (2017)

https://www.cyberdelicsincubator.com (retrieved 10 September 2017)

Emets, E., Rainaud, A., and Gingrich, O. (2016) Analema Group. https://analemagroup.com// (retrieved 5 August 2016).

Grimes, R. L. (2006) Rite out of Place. Oxford University Press, UK.

Haill, L. (2017) Brainwaves Augmenting Consciousness. https://lucianahaill.wordpress.com/ (retrieved 17 October 2017).

Harner, M. (1992) The Way of Shaman. Harper San Francisco, USA.

Levy, M. (1993) Technicians of Ecstasy. Bramble Books, USA.

Maja Murnik, M. (2018) Performing Arts and Virtual Bodies.

Pigott, E., Alter, G., and Marikis, D. (2017) FrequencyBased Light \& Sound Neurotherapy (LSN) Research: A Review of the Research. https://pandorastar.co.uk/ (retrieved 12 October 2017).

Tart, C. T. (1990) Altered States of Consciousness. Harper, New York, USA.

Thomason, T. C. (2018) The Role of Altered States of Consciousness in Native American Healing, Northern Arizona University, Cuyamungue Institute. http://www.cuyamungueinstitute.com/articles-andnews/the-role-of-altered-states-of-consciousness-innative-american-healing/ (retrieved 7 April 2018).

Weinel, J. (2012) Altered states of consciousness as an adaptive principle for composing electroacoustic music [PhD thesis]. http://www.jonweinel.com/media/ASC Commentary 38.p df (retrieved 7 April 2018). 